\title{
Effects of spectacles and telescopes on visual function in students with oculocutaneous albinism
}

\author{
Siddeeqa Jhetam, Khathutshelo P Mashige
}

\begin{abstract}
Discipline of Optometry, School of Health Science, University of KwaZulu-Natal, Private Bag X54001, Durban, 4000 South Africa
\end{abstract}

\begin{abstract}
Purpose: To investigate the effects of spectacle and telescope corrections on visual acuity (VA), contrast sensitivity (CS) and reading rates (RR) in students with oculocutaneous albinism (OCA).

Methods: An observational study design was conducted on 81 students with OCA. Distance and near VA, CS and RR were measured without correction, with spectacle correction and with a combination of spectacle correction and telescopes.

Results: The mean distance and near VA values with a combination of spectacle correction and telescopes were significantly better than those without correction and with spectacle correction alone $(p=0.01)$. Mean CS values achieved with spectacles alone were significantly better than those obtained with a combination of spectacles and telescopes $(p=0.01)$. There was no significant difference between $\log C S$ values obtained without correction compared to those obtained with a combination of spectacle correction and telescopes. There were no significant differences between $\mathrm{RR}$ values obtained with a combination of spectacles and telescopes and those without and with spectacle correction alone (all $\mathrm{p}>0.05$ ).

Conclusion: This article provides valuable information to eye care practitioners on the effects of spectacles and telescopes on visual acuity, contrast sensitivity and reading rate in students with OCA.

Keywords: Oculocutaneous albinism; visual acuity; telescope; contrast sensitivity; reading rate.

DOI: https://doi.org/10.4314/ahs.v20i2.28

Cite as: Jhetam S, Mashige KP. Effects of spectacles and telescopes on visual function in students with oculocutaneous albinism. Afri Health Sci. 2020; 20(2): 758-767. https://doi.org/10.4314/abs.v20i2.28
\end{abstract}

\section{Introduction}

Albinism is a genetic disorder that results from a reduced production of pigment in the skin, eyes and hair. ${ }^{1}$ Individuals with albinism present with reduced visual acuity (VA) and usually experience difficulty in performing activities of daily living such as reading. However, optical and non-optical assistive devices, including but not limited to, spectacles, magnifiers, lighting variations and contrast enhancers, can significantly improve their functional vision. This may be expected as spectacles improve vision by correcting refractive error, while telescopes improve vision by magnifying the images of objects to a size that the individual isable to perceive.

\section{Corresponding author: \\ Khathutshelo P Mashige, \\ Discipline of Optometry, School of Health Science, University of KwaZulu-Natal, Private Bag X54001, Durban, 4000 South Africa \\ Tel: +27312607896 \\ Fax: 0865810908 \\ E-mail: mashigek@ukzn.ac.za}

A VA of $20 / 40(6 / 12)(0.3 \log M A R)$ is assumed to be sufficient for performing most tasks at distance, ${ }^{2,3}$ while a print size of 20/50 (6/15) (1M) (0.4 logMAR) at near is generally used as a goal VA in determining near magnification. ${ }^{4}$ However, it may be possible for patients to achieve better distance and near VA values than the above-mentioned goal VA values with the use of assistive devices.

Contrast sensitivity (CS) can impact a student's daily school activities, such as reading and copying from the chalkboard. Due to the impact on functional vision, magnification alone may not be sufficient to assist students in their daily activities. In addition, students with low vision have been reported to have lower silent reading speeds than normal sighted students of similar grades, which can result in more time spent in attempting to complete a task. ${ }^{5}$

The effects of spectacle correction on $\mathrm{VA}^{6}$ and those of telescopes on functional vision ${ }^{7}$ in persons with albinism have been described before, however, there is a paucity of studies that have evaluated and quantified 
the effects of these devices on important visual function. The current study aims to investigate the effects of spectacles and telescopes on VA, CS and reading rate $(\mathrm{RR})$ in students with oculocutaneous albinism (OCA).

\section{Subjects and methods}

This was an observational study design utilising convenience sampling conducted among students with OCA. The students were from three special education schools namely; Open Air, Ethembeni and Arthur Blaxall Schools, located in the Province of KwaZulu-Natal, South Africa. Eighty one students who were enrolled at the schools returned the consent and assent forms and were included in the study. Ethical approval to conduct the study was obtained from the Biomedical Research Ethics Committee of the University of KwaZulu-Natal (reference: BFC054/14). Permission to conduct the study was also obtained from the relevant statutory bodies and schools.

A comprehensive ocular examination was conducted on all students which included patient history, VA measurements, ocular motility tests, binocular vision, visual fields, CS, RR and objective (retinoscopy) and then subjective refractions. Ophthalmoscopy, slit lamp biomicroscopy and pupil reactions to light were also performed. Uncorrected VA at distance $(4 \mathrm{~m})$ and near $(40 \mathrm{~cm})$ were measured for the right, left and both eyes using $\log M A R$ VA charts. If the student was unable to see the top row of letters, the viewing distance was reduced using appropriate logMAR steps. The test distance, logMAR line VA and equivalent Snellen VA were then recorded and appropriate $\log \mathrm{MAR}$ VA conversions were applied where necessary.

Refractive error was measured objectively using a retinoscope, and then refined subjectively with a trial frame and lenses to obtain the final spectacle prescription. Spectacles aided distance and near VA for the right, left and both eyes were then recorded. Contrast sensitivity was assessed using the Mars Letter Contrast Sensitivity Test placed $50 \mathrm{~cm}$ from the student and appropriate illumination was recorded using a light meter. The lowest contrast level seen with and without the spectacle prescription was determined for the right, left and both eyes.

The RR without and with spectacle correction was assessed by using a RR chart with the appropriate letter size optotype (one line above best VA) placed $40 \mathrm{~cm}$ from the student. The RR was calculated using the formula provided by the chart developers: ${ }^{8}$
Reading rate $=$ number of words read - errors

60 seconds

All the students were provided with training on the use of telescopes, as well as on general aspects of the device such as familiarisation, localisation, focusing and tracing.

Visual acuities with a combination of spectacle prescription and telescopes were also obtained using distance and near logMAR VA charts placed $4 \mathrm{~m}$ and 40 $\mathrm{cm}$ respectively, from the student. The student was asked to hold the monocular telescope in front of the spectacle prescription for the eye being tested with the fellow eye occluded and instructed to look through both the telescope and spectacle prescription in the trial frame simultaneously. The examiner initially focused the telescope subjectively thereafter, the student was requested to refine the focus of the telescope and fixate on the VA chart. The smallest print size read with the combination of spectacle correction and telescopes was determined. For the purpose of this study, VA values of $0.04 \operatorname{logMAR}$ were considered to be equivalent to $0.0 \log$ MAR at both $4 \mathrm{~m}$ and $40 \mathrm{~cm}$ as $0.04 \log$ MAR indicates more than half the number of optotypes were read correctly. Monocular telescopes of varying magnification were used to determine if $0.04 \log$ MAR VA was attainable. Where applicable, binocular telescopes were used and the procedure followed was similar to the one used for monocular testing. However, the focusing distance for near was $25 \mathrm{~cm}$ and after monocular focusing of the device, when both eyes were open, the telescopes were refocused for each eye.

The Mars Letter Contrast Sensitivity Test was used to obtain the lowest contrast level seen with a combination of the spectacle prescription and telescopes. Monocular and binocular (where appropriate) values were determined and the chart was placed at $50 \mathrm{~cm}$ and $25 \mathrm{~cm}$ respectively according to the focus range of the telescopes. Appropriate logCS adjustments were applied in the case of binocular measurements. The distance spectacle prescription was used with the monocular and/ or binocular telescopes that provided the best vision at near or the telescope which provided the best subjective appreciation of the $0.4 \log$ MAR optotype chart.

The RR for each and/or both eyes were determined with the combination of the distance spectacle prescription and monocular and/or binocular telescopes that provided the best vision at near or the telescope which provided the best subjective appreciation of the $0.40 \log$ MAR optotype chart. The RR chart with the 
line above best VA achieved with the combination of spectacles and telescopes, was chosen for students with $0.40 \log$ MAR or worse VA, while the $0.40 \log$ MAR reading rate chart was used for students with best VA better than $0.40 \log$ MAR.

The data was captured into the Statistical Package for Social Sciences (SPSS) version 24 which was also used for statistical analyses. The data was tested for normality with the Kolmogorov-Smirnov test. The $\mathrm{p}$ value of the Kolmogorov-Smirnov test was greater than 0.05, indicating that the data was normally distributed. Pre and post intervention data was analysed using the paired t-test. A probability value of $<0.05$ was considered statistically significant. Analysis of variance (ANOVA) was done when the means of three or more group means were compared. Categorical data was analysed by Pearson chi-square tests or Fisher's exact tests where appropriate.

\section{Results}

There were approximately 100 students with OCA in the three schools, who met the age requirements for the study, and $81(81 \%)$ students including 48 (59.3\%) females and $33(40.7 \%)$ males participated. Their ages ranged from 8 to 21 years (mean $=13.70+3.42$ years).
Distance and near VAs, as well as CS values measured with a combination of spectacle correction and telescopes were not significantly associated with the age of students (all $\mathrm{p}>0.05)$.

Forty three $(53.1 \%)$ students reported having a history of prior use of telescopes, $37(45.7 \%)$ reported not having used telescopes before and $1(1.2 \%)$ did not respond to this question. Distance VA measured for the right eye (RE) with a combination of spectacle correction and telescopes showed a statistically significant relationship $(p=0.04)$ with the prior use of telescopes. However, all other VAs at near and distance, CS values and RR achieved with a combination of spectacle correction and telescopes yielded insignificant relationships with the previous use of telescopes.

Monocular distance VA measured with a combination of spectacle correction and telescopes ranged from $-0.10 \log$ MAR to $0.70 \log$ MAR while binocular VA ranged from $0.00 \log$ MAR to $0.20 \log$ MAR (Table 1). The highest magnification available for distance binocular telescopes was 4X. A calculated magnification of higher than $4 \mathrm{X}$ provided VA of $0.04 \log \mathrm{MAR}$ and better in many of the studnts, thus few students were tested binocularly.

Table 1: Distance logMAR VA

\begin{tabular}{|c|c|c|c|c|c|}
\hline & & $\mathbf{N}$ & $\begin{array}{l}\text { Minimum } \\
(\log M A R)\end{array}$ & $\begin{array}{r}\text { Maximum } \\
(\log M A R)\end{array}$ & $\begin{array}{c}\text { Mean } \\
(\operatorname{logMAR})\end{array}$ \\
\hline \multirow[t]{3}{*}{$\mathrm{RE}$} & Uncorrected & 81 & 0.52 & 1.40 & $0.93+0.18$ \\
\hline & Spectacle correction & 80 & 0.50 & 1.06 & $0.78+0.13$ \\
\hline & $\begin{array}{l}\text { Combination of spectacle } \\
\text { correction and telescopes }\end{array}$ & 79 & -0.10 & 0.70 & $0.06+0.14$ \\
\hline \multirow[t]{3}{*}{ LE } & Uncorrected & 81 & 0.50 & 1.32 & $0.89+0.18$ \\
\hline & Spectacle correction & 79 & 0.50 & 1.10 & $0.74+0.13$ \\
\hline & $\begin{array}{l}\text { Combination of spectacle } \\
\text { correction and telescopes }\end{array}$ & 79 & -0.10 & 0.70 & $0.05+0.13$ \\
\hline \multirow[t]{3}{*}{$\mathrm{BE}$} & Uncorrected & 32 & 0.44 & 1.12 & $0.80+0.15$ \\
\hline & Spectacle correction & 32 & 0.40 & 1.00 & $0.69+0.14$ \\
\hline & $\begin{array}{l}\text { Combination of spectacle } \\
\text { correction and telescopes }\end{array}$ & 5 & 0.00 & 0.20 & $0.08+0.09$ \\
\hline
\end{tabular}

RE, Right eye; LE, Left eye; BE, Both eyes 
The mean differences between distance VA values obtained with the combination of spectacle correction and telescopes and values obtained without correction was $0.86+0.18 \log$ MAR in the right eye and $0.83+$ $0.18 \log M A R$ in the left eye. The mean differences between the distance VA values obtained with the combination of spectacles and telescopes and values obtained with spectacles alone was $0.71+0.15 \log$ MAR and 0.70 $+0.15 \operatorname{logMAR}$ in the right and left eyes respectively. The mean VA obtained with the combinaion of spectacle correction and telescopes was significantly better ( $\mathrm{p}$ $=0.01)$ than VA obtained without spectacle correction and with spectacle correction alone.
Near VA values obtained with a combination of spectacle correction and telescope prescriptions ranged between $-0.04 \log$ MAR and $0.72 \log$ MAR (Table 2). The mean VA achieved binocularly $(0.33 \log$ MAR +0.31$)$ was worse than that achieved monocularly (RE: 0.14 $\log$ MAR + 0.18; LE: 0.13 logMAR + 0.18). The range of available binocular telescopes for near vision used in the study was $2.5 \mathrm{X}$ to $4 \mathrm{X}$. The calculated magnification required to obtain $0.04 \log \mathrm{MAR}$ or better was greater than $4 \mathrm{X}$ for all students, as a result few students were tested binocularly.

Table 2: Near $\log$ MAR VA $(40 \mathrm{~cm}$ and $25 \mathrm{~cm})$

\begin{tabular}{|c|c|c|c|c|c|}
\hline & & $\mathbf{N}$ & $\begin{array}{l}\text { Minimum } \\
(\log M A R)\end{array}$ & $\begin{array}{l}\text { Maximum } \\
(\log M A R)\end{array}$ & $\begin{array}{c}\text { Mean } \\
(\log \text { MAR })\end{array}$ \\
\hline \multirow[t]{3}{*}{ RE } & Uncorrected & 80 & 0.50 & 1.30 & $0.87+0.17$ \\
\hline & Spectacle correction & 79 & 0.40 & 1.08 & $0.76+0.15$ \\
\hline & $\begin{array}{l}\text { Combination of spectacle } \\
\text { correction and telescopes }\end{array}$ & 78 & -0.02 & 0.60 & $0.14+0.18$ \\
\hline \multirow[t]{3}{*}{ LE } & Uncorrected & 80 & 0.40 & 1.30 & $0.84+0.17$ \\
\hline & Spectacle correction & 78 & 0.50 & 1.10 & $0.74+0.13$ \\
\hline & $\begin{array}{l}\text { Combination of spectacle } \\
\text { correction and telescopes }\end{array}$ & 79 & -0.04 & 0.72 & $0.13+0.18$ \\
\hline \multirow[t]{3}{*}{$\mathrm{BE}$} & Uncorrected & 29 & 0.30 & 1.02 & $0.71+0.18$ \\
\hline & Spectacle correction & 29 & 0.30 & 0.92 & $0.65+0.16$ \\
\hline & $\begin{array}{l}\text { Combination of spectacle } \\
\text { correction and telescopes (at } \\
25 \mathrm{~cm} \text { ) }\end{array}$ & 3 & $\begin{array}{c}0.00 \\
\text { (Converted } \\
\text { to } 40 \mathrm{~cm} \text { ) } \\
\end{array}$ & $\begin{array}{c}0.60 \\
\text { (Converted } \\
\text { to } 40 \mathrm{~cm} \text { ) } \\
\end{array}$ & $0.33+0.31$ \\
\hline
\end{tabular}

RE, Right eye; LE, Left eye; BE, Both eyes

The combination of spectacle correction and telescopes significantly improved near VA when compared to values obtained without spectacle correction and with spectacle correction only $(p=0.01)$. The mean differences for near VA with a combination of spectacle correction and telescopes and without correction was $0.72+0.18 \operatorname{logMAR}$ and $0.71+0.19 \log$ MAR for the right and left eyes respectively. The mean differences for the near VA with the combination of spectacle correction and telescopes and VA obtained with spectacle correction was $0.62+0.17 \log$ MAR in the right eye and $0.62+0.18 \log$ MAR in the left eye. Few binocular VA measurements were obtained and were thus not included in the analysis.
Sixty (74.1\%) and $64(79 \%)$ students achieved a distance logMAR acuity of 0.04 or better with a combination of spectacle correction and telescopes in the right eye and left eyes respectively (Table 3). Majority of the students (RE: $51.67 \%$ and LE: $53.13 \%$ ), who achieved 0.04 logMAR or better at distance used a combination of spectacle correction and a 6X telescope, showing that the 6X magnification yielded best VA of $0.04 \log M A R$ or better. When tested binocularly, two of five students also obtained VA of 0.04 or better. The uncorrected distance VA in the right and left eyes were significantly better for those who achieved $0.04 \log$ MAR or better compared to those who did not $(\mathrm{p}=0.01$ and 0.04 respectively). 
With a combination of spectacle correction and telescopes, $43(53.1 \%)$ students were able to achieve a near $\operatorname{logMAR}$ VA of 0.04 or better in the right eye, while $45(55.6 \%)$ achieved the same VA in the left eye (Table $3)$. Hereto, the combination of the spectacle correction and 6X telescope yielded the best VA of $0.04 \log$ MAR or better in many right and left eyes (44.2\% and $48.9 \%)$. One $(1.2 \%)$ of three students tested binocularly also achieved a VA of $0.04 \log$ MAR or better. Uncorrected near VA in the right and left eyes were significantly better for those who achieved $0.04 \log$ MAR or better at distance compared to those who did not $(\mathrm{p}=0.02$ and 0.01 , respectively).

Table 3: Achievement of $0.04 \log M A R$ VA or better at distance and near

\begin{tabular}{|c|c|c|c|c|c|c|c|c|c|c|}
\hline & & \multicolumn{3}{|c|}{$\mathbf{R E}$} & \multicolumn{3}{|c|}{ LE } & \multicolumn{3}{|c|}{$\mathrm{BE}$} \\
\hline & & Number & (N) & $\begin{array}{c}\text { Percentage } \\
(\%) \\
\end{array}$ & Number & (N) & $\begin{array}{c}\text { Percentage } \\
(\%) \\
\end{array}$ & Number & (N) & $\begin{array}{c}\text { Percentage } \\
(\%) \\
\end{array}$ \\
\hline \multirow[t]{5}{*}{ Distance } & Achieved & 60 & & 74.1 & 64 & & 79 & 2 & & 2.5 \\
\hline & $\begin{array}{l}\text { Did not } \\
\text { achieve }\end{array}$ & 19 & & 23.5 & 15 & & 18.5 & 3 & & 3.7 \\
\hline & Total & 79 & & 97.5 & 79 & & 97.5 & 5 & & 6.2 \\
\hline & Missing & 2 & & 2.5 & 2 & & 2.5 & 76 & & 93.8 \\
\hline & Total & 81 & & 100 & 81 & & 100 & 81 & & 100 \\
\hline \multirow[t]{5}{*}{ Near $(40 \mathrm{~cm})^{*}$} & Achieved & 43 & & 53.1 & 45 & & 55.6 & 1 & & 1.2 \\
\hline & $\begin{array}{l}\text { Did not } \\
\text { achieve }\end{array}$ & 35 & & 43.2 & 34 & & 42 & 2 & & 2.5 \\
\hline & Total & 78 & & 96.3 & 79 & & 97.5 & 3 & & 3.7 \\
\hline & Missing & 3 & & 3.7 & 2 & & 2.5 & 78 & & 96.3 \\
\hline & Total & 81 & & 100 & 81 & & 100 & 81 & & 100 \\
\hline
\end{tabular}

*BE VA measurements conducted at $25 \mathrm{~cm}$ and converted to $40 \mathrm{~cm} \log M A R$ values

Forty one students achieved a VA of $0.04 \log \mathrm{MAR}$ or better at $40 \mathrm{~cm}$ with the right eye. Of those, 38 (92.7\%) achieved a VA of $0.04 \operatorname{logMAR}$ or better at distance. Thirty five students did not achieve a VA of $0.04 \mathrm{log}$ MAR or better at $40 \mathrm{~cm}$ with the right eye, however, of these students, twenty (57.1\%) achieved $0.04 \log$ MAR at distance, and this was statistically significant (Pearson chi-square $=13.195, \mathrm{p}=0.01)$. Forty three students achieved a VA of $0.04 \log M A R$ or better at $40 \mathrm{~cm}$ with the left eye. Of those, $41(95.3 \%)$ achieved a distance
VA of $0.04 \log M A R$ or better. Thirty four students did not achieve $0.04 \operatorname{logMAR}$ or better at $40 \mathrm{~cm}$, however, of these $21(61.8 \%)$ achieved a distance VA of 0.04 $\operatorname{logMAR}$ or better, and this was statistically significant (Pearson chi-square $=13.653, \mathrm{p}=0.01$ ).

The mean $\log C S$ measured with a combination of spectacle correction and telescopes was similar for the right and left eyes $(1.39+0.24 \log \mathrm{CS}, 1.38+0.25 \log \mathrm{CS}$, respectively) (Table 4). 
Table 4: Contrast sensitivity $(\log C S)$

\begin{tabular}{cccccc}
\hline & & $\begin{array}{c}\text { Number } \\
(\mathbf{N})\end{array}$ & $\begin{array}{c}\text { Minimum } \\
(\mathbf{l o g} C S)\end{array}$ & $\begin{array}{c}\text { Maximum } \\
(\mathbf{l o g C S})\end{array}$ & $\begin{array}{c}\text { Mean } \\
(\mathbf{l o g C S})\end{array}$ \\
\hline \multirow{2}{*}{$\mathrm{RE} \quad$} & 80 & 0.56 & 1.88 & $1.43+0.26$ \\
& Uncorrected & 79 & 0.88 & 1.88 & $1.52+0.24$ \\
& Spectacle correction & 79 & 0.92 & 1.88 & $1.39+0.24$ \\
& Combination of spectacle & & & & \\
& correction and telescopes & & & & \\
& Uncorrected & 80 & 0.48 & 1.92 & $1.42+0.33$ \\
& Spectacle correction & 78 & 0.92 & 1.92 & $1.53+0.25$ \\
& Combination of spectacle & 76 & 0.76 & 1.88 & $1.38+0.25$ \\
& correction and telescopes & & & & \\
\hline
\end{tabular}

RE, Right eye; LE, Left eye

Moderate loss of CS was the most common classification for monocular tests. No statistically significant differences were found between CS values (mean difference RE: $-0.04+0.27 \log \mathrm{CS}$, LE: $-0.04+0.30$ $\log \mathrm{CS}$ ) obtained with the combination of spectacle correction and telescopes and without correction. Contrast sensitivity values obtained with spectacle correction only were significantly higher than those obtained with a combination of spectacle correction and telescopes $(\mathrm{p}=0.01)$. The mean CS difference in the right and left eyes were $-0.14+0.22 \log C S$ and $-0.17+0.24$ $\log \mathrm{CS}$ respectively. The highest mean $\log \mathrm{CS}$ (RE: 1.37
+0.25 , LE: $1.35+0.27$ ) values were obtained using a combination of a $3 \mathrm{X}$ telescope and the relevant spectacle correction. The lowest mean values (RE: $0.91+$ $0.27 \log C S$, LE: $0.91+0.29 \log$ CS) were obtained using an $8 \mathrm{X}$ telescope with the relevant spectacle correction. Contrast sensitivity decreased with an increase in magnification.

The RR (wpm) with a combination of spectacle correction and telescopes ranged from 14 to 116 words per minute, the mean and standard deviation being $64.31+$ 25 words per minute (Table 5).

Table 5: Minimum, maximum and mean reading rate values

\begin{tabular}{ccccc}
\hline & $\begin{array}{c}\text { Number } \\
(\mathbf{N})\end{array}$ & $\begin{array}{c}\text { Minimum } \\
\text { (words per minute) }\end{array}$ & $\begin{array}{c}\text { Maximum } \\
\text { (words per minute) }\end{array}$ & $\begin{array}{c}\text { Mean (words per } \\
\text { minute) }\end{array}$ \\
\hline Uncorrected & 65 & 21 & 140 & $64.58+26.61$ \\
Spectacle correction & 61 & 7 & 119 & $62.20+25.66$ \\
Combination of spectacle & 61 & 14 & 116 & $64.31+25.00$ \\
correction and telescopes & & & & \\
\hline
\end{tabular}


The mean difference in $R R$ values obtained with a combination of spectacles and telescopes and without correction was $-1.26+22.67 \mathrm{wpm}$, while the mean difference for values obtained with a combination of spectacles and telescopes and spectacle correction was $-0.70+26.58 \mathrm{wpm}$. There was no statistically significant difference between these variables $(p=0.67$ and $\mathrm{p}=0.84$ respectively). The relationship between $\mathrm{RR}$ achieved with a combination of spectacle correction and telescopes was positively and significantly associated with age $(p=0.01)$. The mean $R R$ for those with prior use of devices and those without prior use were $72.49+21.78$ and $53.31 \pm 25.20$ words per minute respectively. There was a statistically significant difference between these two variables $(p=0.01)$. The association between telescope magnification and the RR achieved with a combination of spectacle correction and telescopes was statistically significant $(p=0.04)$. However, telescopic near VA and the RR achieved with a combination of spectacle correction and telescopes were not statistically significant $(\mathrm{p}=0.10)$. There was no significant relationship between telescopic magnification and telescopic near VA $(\mathrm{p}=0.88)$.

\section{Discussion}

This study aimed at investigating the effects of spectacles and telescopes on VA, CS and RR. A combination of spectacle correction and telescopes significantly improved distance and near VA. Mean CS values achieved with spectacles alone were significantly better than those achieved with a combination of spectacles and telescopes. However, the RR did not significantly improve with a combination of spectacle correction and telescopes.

Mean VA values achieved with a combination of spectacle correction and telescopes were better than those reported in other studies involving similar subjects.9,10,11 For example, Pereira et al9 reported an average distance VA of $0.70 \operatorname{logMAR}$, which was worse than that reported by $\mathrm{Oh}$ et al10 (0.3 logMAR) and Schwering et al ${ }^{11}$ (0.3 $\log$ MAR). Oh et al10 reported the mean distance VA with telescopes to be $0.3 \log$ MAR, the prescribed telescope was determined based on the participants' satisfaction with vision. Schwering et al11 recommended telescopes to 115 participants who achieved 0.3 logMAR. These studies showed that telescopes improved vision, however, both studies did not use a goal VA of $0.04 \log$ MAR thus it is difficult to conclude that the achieved VA was the best possible that could be ob- tained. Similarly, Sacharowitz ${ }^{12}$ reported distance vision improvement with the aid of telescopes in all 40 participants at a university based eye clinic in South Africa. However, the author did not report to what VA level the vision improved. In the current study, magnification was increased in a clinical environment to achieve the goal VA of $0.04 \log$ MAR or better, the difference in the goal VA for the different studies could account for the high difference achieved for the mean distance VA.

In summary, students with spectacle correction alone achieved distance VA that fell into the category of "severe or moderate visual impairment classification." 13 However, the resultant VA with the addition of the telescope fell into the "moderate to no visual impairment classification".

A combination of telescopes and spectacle correction significantly improved vision to "normal" levels in students with OCA as the majority (70\%) achieved monocular distance VA of $0.04 \operatorname{logMAR}$ or better. Common problems related to distance vision at school reported by students with visual impairment in a study conducted by Ganesh et $\mathrm{al}^{14}$ included transcribing from the blackboard and locating objects that have fallen to the ground. Various factors may lead to such difficulties including the size of written text on the board or the size of the dropped object. The current study show/ span $>$ ed that with increased telescopic magnification, students with OCA can achieve "normal vision" of $0.04 \log$ MAR and can therefore, enroll in mainstream schools. Studies ${ }^{15,16}$ suggested that clear and comfortable vision indicated by good VA is important to ensure the ability of children to learn. Their vision can be improved which will lead to transcribing from the board and locating fallen items easier to accomplish. Ganesh et al14 also highlighted that students with OCA or visual impairment reported that the numbers and details on buses were difficult to see. The improved vision achieved by students with albinism in this study using a combination of spectacle correction and telescopes is at least $0.04 \log$ MAR. This can allow them to see the numbers and details on buses and therefore improve and facilitate access to public transport. Furthermore, vision problems in children with albinism have been reported to cause developmental difficulties and have been linked to anti-social behavior. ${ }^{15,16}$ Therefore, an improvement of vision achieved by a combination of spectacle correction and telescopes can lead to better educational and general quality of life outcomes in persons with this condition. 
The better near VA achieved with a combination of spectacle correction and telescopes compared to spectacle correction only, suggests that this combination can enhance the students' ability to conduct near visual tasks and related activities. Therefore, important school activities such as reading magazines and books can be achieved when students with OCA are corrected with this combination.

Various forms of magnification can be used for near tasks by students at schools. Many of these magnifiers have close working distance and a common problem reported by students with low vision is reading books at arm's length. ${ }^{14}$ The monocular telescopes used in this study provided a reading distance of $40 \mathrm{~cm}$. The combination of the spectacle correction and telescopes for near reading allowed for a larger working distance and improved vision. The achievement of $0.04 \log$ MAR or better near VA allows for fine print of texts to be seen with more ease than without telescopes. The difficulty experienced with near tasks can be reduced by the use of telescopes which might improve the students' efficiency at school.

Students with sufficient ocular accommodation may use relative distance magnification to achieve better near acuity, however, further assistance can be provided by low vision devices. The continuous use of reading material at a reduced near distance can result in accommodative spasm. ${ }^{17}$ Thus, an appropriate near low vision device such as a telescope can be used to provide an intermediate and comfortable reading position. Unaided distance and near VA for those who achieved "normal levels of vision" with the combination of spectacle correction and telescopes was significantly better than those who did not achieve "normal levels". This implies that uncorrected VA may impact the ability to achieve normal vision with low vision assistive devices. Considering the level of improvement achieved with the combination of spectacle correction and telescopes, eye care practitioners need to prescribe telescopes to enhance vision of OCA students beyond that provided solely by spectacle correction alone. However, caution should be exercised when using magnification for general tasks. For example, with an increase in magnification, the VA may improve but the visual field will reduce, resulting in the ease of use being limited. Such magnification might be appropriate for spotting and tracing tasks such as reading from the board.
A combination of spectacle correction and telescopes resulted in better VA values compared to those with spectacle correction alone $(p=0.01)$. Such a significant change was not seen when CS values without spectacle correction were compared to values obtained with a combination of spectacle correction and telescopes. This study found a reduction in monocular logCS perceived when magnification was increased. The highest perceivable monocular $\log C S$ was achieved with a combination of a $3 \mathrm{X}$ telescope and spectacle correction, however, the lowest was achieved with an $8 \mathrm{X}$ telescope and spectacle correction. This finding may be attributed to the reduction in the field of view associated with magnification, as the letter was enlarged, the student would find difficulty to see the letter in its entirety leading to a misidentification of the letter perceived. Loshin and Browning ${ }^{7}$ reported an increase in CS functions in the horizontal and vertical planes with the use of telescopes and appropriate reading caps. The study by Loshin and Browning ${ }^{7}$ involved grating stimuli while our study used letter stimuli, which are more appropriate to students in schools. It is important for eye care practitioners to verify the impact of the telescope prescribed to students on reading material associated with normal daily functioning.

No significant difference was observed between the RR obtained with and without spectacle correction and those obtained with a combination of spectacle correction and telescopes. The reading rate VA used for the combination of spectacle correction and telescopes was also one line above best VA only in the event the $0.4 \log$ MAR $(1 \mathrm{M})$ chart was not visible with the combination, and therefore low acuity reserve was utilised. Amore et $\mathrm{al}^{18}$ reported that fixation stability impacts reading speed. Unstable fixation was correlated with reduced reading speed despite the use of magnifying devices, thus acuity reserve might not be the only factor that limited the improvement in reading rate with a combination of spectacle correction and telescopes as all but one student had nystagmus.

\section{Limitations}

This study has certain limitations that must be acknowledged. Firstly, the training and practice in the use of telescopes was limited due to time constraints. Secondly, the acuity reserve of one line, was insufficient to allow the student to achieve an efficient RR. Thirdly, CS measurements may have been affected by the choice of stimuli, as letter optotypes rather than grating stimuli were used. Incorrect identification of letters may have 
been reported due to the impact of magnification on the size of the stimuli thereby reducing the field of view of the letter seen.

\section{Implications and applications of findings}

Vision rehabilitation for learners with OCA should include telescope trials as these provide visual acuity improvement despite the presence of nystagmus. As telescopes are listed as part of the minimum equipment requirements by the Health Professions Council of South Africa for low vision assessments, practitioners should have a focusable $6 \mathrm{X}$ telescope for trial on their patients as this achieved maximum improvement in VA for most OCA learners. Clinicians should strive to achieve maximum vision while providing appropriate magnification considering ease of use and visual field restrictions associated with telescopes. Longer training and practice sessions might improve the use and familiarisation of the telescopes. Reading rate should be determined using an acuity reserve and the lowest magnification that provides $1 \mathrm{M}(0.40 \log \mathrm{MAR})$. To determine the effect on CS, grating stimuli may be used, however, one should be cautious with the increase in image size and low spatial frequencies.

\section{Conclusion}

Distance and near VAs of students with OCA improved to $0.04 \log$ MAR with the use of telescopes in combination with spectacle prescription. Contrast sensitivity improved to normal levels in some students with OCA, thus telescopes play an important role in improving VA as well as functional vision in this group. The RR did not significantly improve with telescopes in students with OCA, therefore, clinicians should be aware of the appropriate acuity reserve measurements and trial magnification options to use. Further research is needed to assess the impact of telescopes on specific vision-related tasks, school performance/educational outcomes, psychosocial and quality of life (visual functioning, well-being, headaches and risks of accidents) as well as positive (acaemic, appearance and self-esteem) and negative (academic, teasing and bullying) outcomes following correction.

\section{Acknowledgements}

KPM is a University of KwaZulu-Natal (UKZN) Developing Research Innovation, Localisation and Leadership in South Africa (DRILL) fellow. DRILL, is a NIH D43 grant (D43TW010131) awarded to UKZN in 2015 to support a research training and induction programme for early career academics. The content is solely the responsibility of the authors and does not necessarily represent the official views of DRILL and the National Institutes of Health. The authors thank all the students from the three schools who participated in this study. We also thank Professor Brown for assistance with statistical analyses.

\section{Conflict of interest}

None.

\section{References}

1. Levin AV, Stroh E. Albinism for the busy clinician. JAAPOS. 2011; 15(1): 59-66.

2. Jose RT. Treatment Options. In: Jose RT, editor. Understanding Low Vision. New York: American Foundation for the Blind, 1983; p. 211-248.

3. Williams DR. Low vision distance systems II: Telescopes and telemicroscopes. In: Brilliant RL, editor. Essentials of Low Vision Practice. Woburn: Butterworth-Heinemann, 1999; p. 147-200.

4. Matchinski T, Brilliant RL, Bednarski M. Low vision near systems I: Microscopes and magnifiers. In: Brilliant RL, editor. Essentials of Low Vision Practice. Woburn: Butterworth-Heinemann, 1999; p. 201-237.

5. Corn AL, Wall RS, Jose RT, Bell JK, Wilcox K, Perez A. An initial study of reading and comprehension rates for students who received optical devices. J Vis Impair \& Blindness. 2002; 96(5): 322-334.

6. Oduntan AO, Raliavhegwa M, Lund PM. Vision status of children with oculocutaneous albinism. Ophthal Physiol Opt. 2002; 22(6): 572-573.

7. Loshin DS, Browning RA. Contrast sensitivity in albinotic patients. Am J Optom Physiol Opt. 1983; 6(3): 158-166.

8. Nirghin U. Design, reliability and validity of a paediatric rate of reading chart. Unpublished Masters' Dissertation]. South Africa: University of KwaZulu-Natal; 2012.

9. Pereira DFL, Araujo EL, Patuzzo FVD. Profile of albinism with low vision and improvement of visual acuity with the adaptation of optical and/or electronic resources. Rev Bras De Oftalmol. 2016; 75(6): 456-460.

10. Oh DH, Park SH, Lee JK, Moon NJ. Clinical findings and results of low vision devices in pediatric patients with albinism. J Korean Ophthalmol Soc. 2011; 52(4): 466-471.

11. Schwering MS, Kumar N, Bohrmann D. Msukwa G, Kalua K, Kayange P, et al. Refractive errors, visual impairment, and the use of low-vision devices in albinism in Malawi. Graef Arch Clin Exp Ophthalmol. 2015; 253(4): 655-661. 
12. Sacharowitz HS. An overview of oculocutaneous albinism in South Africa. Paper presented at: The International Conference on Low Vision 1999. Proceedings of the Low Vision Rehabilitation: Assessment, Intervention and Outcomes, International Conference on Low Vision; 1999; New York, USA. 1999. p. 41-46.

13. World Health Organisation (WHO). International Statistical Classification of Diseases and Related Health Problems $10^{\text {th }}$ Revision (ICD-10)-WHO version for 2016. Available from: http://apps.who.int/classifications/icd10/browse/2016/en\#/H54. Accessed on 23/01/2018.

14. Ganesh S, Sethi S, Srivastav S, Chaudhary A, Arora P. Impact of low vision rehabilitation on functional vision performance of children with visual impairment. Oman J Ophthalmol. 2013; 6(3): 170-174.
15. Johnson RA, Zaba JN. The visual screening of adjudicated adolescents. J Behav Optom. 1999; 10(1): 13-17. 16. Cooper JS, Burns CR, Cotter SA, Daum KM, Griffin JR, Scheiman MM. Optometric clinical practice guideline: Care of the patient with accommodative and vergence dysfunction. American Optometric Association, 2001; p. 6. Available from: https://www.aoa.org/ documents/optometrists/CPG-18.pdf. Accessed on 06/07/2018.

17. Barber A, Johnson R, editors. Behavioral aspects of vision care. Santa Ana CA: Optometric Extension Program; 2002; 42: 1-7.

18. Amore FM, Fasciani R, Silvestri V, Crossland MD, de Waure C, Cruciani F, et al. Relationship between fixation stability measured with MP-1 and reading performance. Ophthal Physiol Opt. 2013; 33(5): 611-617. 